\title{
Etude comparative de l'exploitation traditionnelle de la pintade locale (Numida meleagris) dans deux villages, Toêghin et Sambonaye, au Burkina Faso
}

\author{
R. SANFO ${ }^{1 *}$, S. OUOBA IMA ${ }^{2}$, I. SALISSOU ${ }^{3}$ et H. H. TAMBOURA ${ }^{4}$ \\ ${ }^{I}$ Institut de l'Environnement et de Recherches Agricoles (INERA), Direction Régionale de l'Environnement \\ Agricole et de Formation de Kamboinsé, Ouagadougou, Burkina Faso. \\ ${ }^{2}$ Institut de l'Environnement et de Recherches Agricoles (INERA), Direction Régionale de l'Environnement \\ Agricole et, station de Saria, Burkina Faso. \\ ${ }^{3}$ Institut National de la Recherche Agronomique du Niger (INRAN), Niger. \\ ${ }^{4}$ Institut de l'Environnement et de Recherches Agricoles (INERA), Direction Régionale de l'Environnement \\ Agricole et de Formation de Kamboinsé, Ouagadougou, Burkina Faso. \\ *Auteur correspondant, E-mail : r_sanfo@yahoo.fr
}

\section{RESUME}

L'élevage de la pintade se pratique à Sambonaye comme à Toêghin, sans distinction de sexes, respectivement dans les régions du Centre et du Sahel du Burkina Faso. Il comporte dans sa pratique à la fois des avantages et également des inconvénients bien connus des producteurs. Cette activité représente, non seulement une source importante de revenus et de protéines pour la population, mais elle répond également à leurs besoins socioculturels. Les produits de cet élevage servent essentiellement à la vente, à la reproduction, aux dons, à l'autoconsommation et également aux sacrifices. Les différents pourcentages affectés à chacun des éléments d'exploitation sont approchés dans cette étude. Cette activité est liée à la saison, car défavorable en saison sèche, elle est favorable à la saison des pluies. Mais, cet élevage connaît malheureusement des contraintes multifactorielles, parmi lesquelles les contraintes sanitaires qui conduisent à des taux de mortalité très élevés. L'élevage de la pintade revêt plusieurs avantages et inconvénients avec lesquels l'éleveur est obligé de composer tous les jours. Mais les différentes voies d'exploitation de la pintade demeurent variables d'une année à l'autre et d'un village à l'autre.

() 2014 International Formulae Group. All rights reserved.

Mots clés : Burkina Faso, avantages, inconvénients, exploitation, pintades.

\section{INTRODUCTION}

L'élevage de poulet local est largement pratiqué en milieu rural et est l'une des principales activités menées par les personnes disposant de peu de ressources (Vidogbèna et al., 2010). Nous pouvons dire autant pour les pintades, surtout au Burkina Faso. Cette expansion est liée à l'importance de cet élevage, non seulement grâce à sa rentabilité pour la sécurité alimentaire (Nagalo, 1984), mais aussi pour ses produits prisés par la population (viande et œufs) et qui ont été recommandés pour combler l'insuffisance en protéine en raison du cycle de reproduction court de la pintade. L'élevage de la pintade doit également cette expansion au rôle qu'il 
joue dans les domaines économiques et socioculturels.

L'étude a été conduite dans la zone Centre et au Sahel du Burkina Faso, respectivement dans les villages de Toêghin et de Sambonaye.

Le présent article, tout en décrivant les avantages et les inconvénients de l'élevage de la pintade, veut également aborder leur effectif en fonction de la saison et déterminer le pourcentage de pintades et des œufs destinés chacun aux divers composantes de l'exploitation comme à la vente, à la reproduction, à l'autoconsommation et aux sacrifices.

\section{MATERIEL ET METHODES}

Matériel

Les données ont été recueillies à l'aide d'un questionnaire formel élaboré à cet effet. Ce questionnaire était relatif à l'identification $\mathrm{du}$ site et des producteurs, les atouts et les insuffisances de l'élevage, l'élevage en fonction des saisons et aussi l'utilisation de la pintade et de ses œufs en méléagriculture traditionnelle. Des interviews semi structurées ont été conduites dans le but de mieux préciser les informations pertinentes.

\section{Méthodes}

L'étude sur les avantages et les inconvénients liés à la pintade, son élevage en fonction de la saison, de même que l'utilisation de celle-ci et de ses œufs, a été conduite en saison sèche, de mars à avril 2003, dans les deux sites, Toêghin et Sambonaye. Les sites de l'étude, Sambonaye au Nord et Toêghin au Centre, ont été retenus pour leurs importances en élevage de pintades et pour leur collaboration avec l'Institut de l'Environnement et de Recherches Agricoles (INERA). L'étude a porté sur un total de 100 productrices et producteurs par site, tirés au sort et faisant de l'élevage de la pintade une activité majeure.

\section{Analyse statistique}

Les saisies des données et le tracé des histogrammes ont été effectués à l'aide du logiciel Excel (2003) sous Windows. Les analyses de variance et la comparaison des moyennes (présentées sous forme de moyenne \pm écartype) ont été faites à l'aide du logiciel Stat View, version 4.5 au seuil de $5 \%$.

\section{RESULTATS}

Sites d'enquête et Producteurs

Le site de l'enquête au Nord est localisé dans la région du Sahel, de la province du Séno, de la Commune et du Département de Dori, et du village de Sambonaye. Le deuxième site de l'enquête du centre relève de la région du Centre, province du Kadiogo, Commune et du Département de Toêghin (Figure 1).

Les producteurs auxquels a été administré le questionnaire sont au nombre de 74 hommes (74\%) et de 26 femmes (26\%) à Sambonaye âgés en moyenne de 48 ans. A Toêghin, le nombre de producteurs a été de 56 hommes (56\%) et 44 femmes (44\%), d'un âge moyen de 54 ans.

\section{Avantages et inconvénients de l'élevage des pintades \\ Dans le Tableau 1 sont logés les} avantages et les inconvénients majeurs qui meublent la pratique de l'élevage de la pintade selon les producteurs de Toêghin et de Sambonaye. L'élevage de la pintade confère au bénéficiaire un certain prestige financier et social. Face à cette intervention monétaire et à ce prestige, se dresse la mortalité des pintadeaux, qui constitue le premier inconvénient de l'élevage de la pintade à Toêghin et à Sambonaye. 


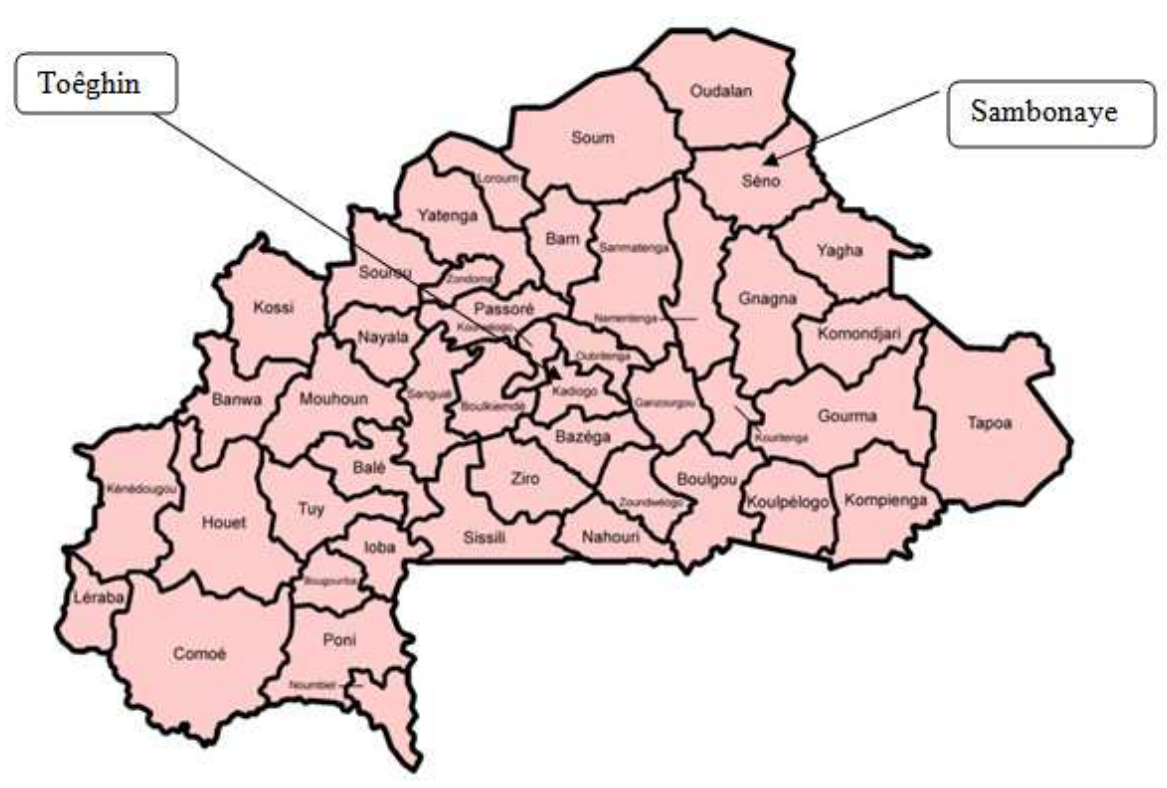

Figure 1 : Localisation des sites de l'étude.

\section{Effectif suivant la saison}

Tant à Sambonaye qu'à Toêghin, les effectifs des pintades varient en fonction des saisons. Elles sont élevées en saison de pluie car les pintades se reproduisent en cette saison, puis baissent en saison sèche à cause des ventes et des mortalités. Les effectifs des pintades et de leurs œufs sont représentés par la Figure 2. Les pintadeaux sont très nombreux en saison humide, mais leur nombre diminue drastiquement en saison sèche. Ceci est en accord avec l'assertion des producteurs quand ils disent que la saison hivernale est défavorable à la survie des pintadeaux. Ceux de Toêghin sont plus nombreux que ceux de Sambonaye, tant en saison humide qu'en saison sèche. Ceci laisse prévoir que ceux de Toêghin interviennent dans l'alimentation des pintades en saison sèche par des compléments.

En saison sèche, la ponte et l'éclosion s'avèrent défavorables alors que la survie des pintadeaux est cependant favorable. En saison hivernale, c'est l'inverse qui est remarqué dans les deux villages. Le Tableau 2 résume les paramètres et leurs causes supposées par les producteurs.

\section{Exploitation}

Chez le producteur, les pintades et leurs œufs sont destinés à la vente, à la mise en reproduction, à l'autoconsommation, aux dons et aux sacrifices.

\section{Ventes}

\section{Prix des oufs et des pintades}

Les prix des œufs est partout le même, les jeunes coûtant plus chers à Sambonaye tandis que les adultes sont plus chers à Toêghin, à cause de sa localisation (non loin de Ouagadougou).

Les prix moyen des œufs est de 50 FCFA $(0,08 \pm 0,00 €)$, celui des jeunes pintades mâles est de 1.467 $\pm 231,25$ FCFA $(2,24 \pm 0,35$ $€)$, celui des jeunes pintades femelles de $1.480 \pm 230,29$ FCFA $(2,75 \pm 0,35 €)$, celui des mâles adultes de 2.119 $\pm 227,4$ FCFA $(3,23 \pm 0,35 €)$ et celui des femelles adultes de 
$2.111 \pm 223,36 \quad$ FCFA $\quad(3,22 \pm 0,34 \quad €) . \quad$ Le Tableau 3 présente les prix pratiqués dans les deux sites.

\section{Nombre d'oufs et de pintades vendus par village}

A Toêghin, les ventes des pintades sur pieds sont plus élevées, tant chez les mâles que chez les femelles. Les pintades mâles ont été vendues à un pourcentage plus élevé à Toêghin qu'à Sambonaye qui a connu par contre un pourcentage plus élevé de vente d'œufs.

A Sambonaye, c'est l'organisation des producteurs en équipe pour la vente des œufs qui montre en partie le pourcentage d'œufs plus élevé que ceux de Toêghin. En période d'hivernage, les œufs sont en effet récoltés pendant la semaine et transportés à Dori chaque vendredi pour la commercialisation.

Avec l'avancée de la saison hivernale et la rareté des œufs, ceux-ci se vendent à 55 FCFA l'unité dans les deux sites. La vente constitue la première voie de sortie, donc le premier objectif de l'élevage de la pintade et de ses œufs, tant à Sambonaye qu'à Toêghin. La Figure 3 schématise le nombre de pintades vendues en pourcentage.

\section{Reproduction}

Les producteurs assurent la reconstitution de leur cheptel essentiellement par la reproduction. A Sambonaye, les pintades mâles et femelles introduits à la reproduction ont un pourcentage plus élevé qu'à Toêghin. Les mâles ont des pourcentages moins élevés que les femelles dans les deux villages. Dans le rapport des pourcentages, on observe qu'il faut 1 mâle contre 2,2 femelles à Sambonaye et 1 mâle contre 2,25 femelles à Toêghin. Ces chiffres représentent ainsi les sex-ratios dans chacun des deux villages. L'introduction à la reproduction constitue le deuxième objectif de l'élevage de la pintade. La Figure 4 présente le pourcentage de pintades et de leurs œufs mis à la reproduction à Sambonaye et à Toêghin.

\section{Autoconsommation}

Ceux de Sambonaye consomment beaucoup plus de pintades mâles que les pintades femelles dans une proportion de 2,87 tandis que ceux de Toêghin en consomment dans une proportion de 1,25. Les habitants de Sambonaye consomment les pintades sur pieds plus que ceux de Dori. Ceux de Toêghin consomment par contre plus d'œufs que ceux de Sambonaye. Le pourcentage de pintades et de leurs œufs est présenté par la Figure 5.

\section{Dons}

Les producteurs de Sambonaye et de Toêghin donnent plus les pintades mâles à leurs visiteurs que les pintades femelles. Ceux de Sambonaye essentiellement des pintades mâles sur pieds, tandis qu'à Toêghin, ils donnent surtout des œufs. Ceci montre que les œufs de pintades jouent un rôle prépondérant dans les rapports sociaux qui lient le producteur aux personnes étrangères. $\mathrm{La}$ Figure 6 présente le pourcentage de pintades et des œufs donnés par les producteurs dans les deux sites.

\section{Sacrifices}

Les producteurs de Sambonaye ne font pas de sacrifices avec la pintade sur pieds ni avec ses œufs. Ceux de Toêghin utilisent trois fois plus des pintades femelles pour les sacrifices que les pintades mâles et leurs œufs. La Figure 7 donne le pourcentage de pintades exploitées en sacrifices dans les deux villages. 
Tableau 1 : Avantages et inconvénients liés à l'élevage de la pintade à Sambonaye et à Toêghin.

\begin{tabular}{ll}
\hline Avantages & Inconvénients \\
\hline $\begin{array}{l}\text { Source de revenus monétaires pour subvenir aux } \\
\text { besoins de la famille et sources de protéines } \\
\text { pour les éleveurs. }\end{array}$ & $\begin{array}{l}\text { Taux très élevé de mortalité des pintadeaux, sans } \\
\text { signes préalables, méconnaissance de produits } \\
\text { appropriés. }\end{array}$ \\
\hline $\begin{array}{l}\text { Aisance dans la commercialisation de la pintade } \\
\text { et de ses œufs. }\end{array}$ & $\begin{array}{l}\text { Pintades devenant sauvages quand ils sont } \\
\text { brutalisés. }\end{array}$ \\
\hline Viande de la pintade agréable à consommer. & $\begin{array}{l}\text { Existence de conflits entre les éleveurs au } \\
\text { moment des pontes (si jamais elles pondent } \\
\text { ensemble). }\end{array}$ \\
\hline Multiplication rapide. & Risque pour les pintades de devenir sauvages. \\
\hline $\begin{array}{l}\text { Double avantages car donne de la viande et des } \\
\text { œufs. }\end{array}$ & Pintades n'aimant pas les coins insalubres. \\
\hline Eufs très sollicités. & Prédation. \\
\hline Pintades résistantes à l'âge adulte. & $\begin{array}{l}\text { Vols des oufs surtout et de pintades fréquents à } \\
\text { Sambonaye. }\end{array}$ \\
\hline $\begin{array}{l}\text { Elevage facile car pas exigeant en } \\
\text { investissements majeurs. }\end{array}$ & \\
\hline
\end{tabular}

Tableau 2 : L'élevage des pintadeaux et des pintades en fonction des saisons.

\begin{tabular}{|c|c|c|c|}
\hline Saison & Paramètre & Etat & Cause \\
\hline & Ponte & Défavorable & $\begin{array}{l}\text { Alimentation pauvre quantitativement et } \\
\text { qualitativement (absence d'insectes, de } \\
\text { termites, de vers, etc.) }\end{array}$ \\
\hline \multirow[t]{3}{*}{$\begin{array}{l}\text { Saison } \\
\text { sèche }\end{array}$} & Eclosion & Défavorable & $\begin{array}{l}\text { Chaleur excessive entraînant la poule à } \\
\text { abandonner les œufs de temps en temps. }\end{array}$ \\
\hline & Survie pintadeaux & Favorable & $\begin{array}{l}\text { Les pintadeaux ne souffrent pas du froid, de } \\
\text { la rosée, de l'humidité. }\end{array}$ \\
\hline & Ponte & Favorable & $\begin{array}{l}\text { Abondance d'aliment (termites, vers, } \\
\text { insectes, verdures, etc.) et humidité. }\end{array}$ \\
\hline \multirow[t]{2}{*}{$\begin{array}{l}\text { Saison } \\
\text { hivernale }\end{array}$} & Eclosion & Favorable & $\begin{array}{l}\text { Il fait relativement frais et la poule } \\
\text { n'abandonne pas les œufs. }\end{array}$ \\
\hline & Survie pintadeaux & Défavorable & $\begin{array}{l}\text { Les pintadeaux qui sont thermosensibles } \\
\text { souffrent du froid et de l'humidité. }\end{array}$ \\
\hline
\end{tabular}

Tableau 3 : Prix de vente de la pintade et de ses œufs à Sambonaye et à Toêghin.

\begin{tabular}{lccccc}
\hline Désignation & EUuf & \multicolumn{2}{c}{ Jeunes } & \multicolumn{2}{c}{ Adultes } \\
\cline { 3 - 6 } & & Mâles & Femelles & Mâles & Femelles \\
\cline { 3 - 6 } & & $1.797 \pm 274,76$ & $1.828 \pm 277,36$ & $2.041 \pm 189,54$ & $2.058 \pm 205,14$ \\
Sambonaye & $50 \pm 0,00$ & $1.132 \pm 183,21$ & $2.197 \pm 265,26$ & $2.163 \pm 241,58$ \\
\hline
\end{tabular}




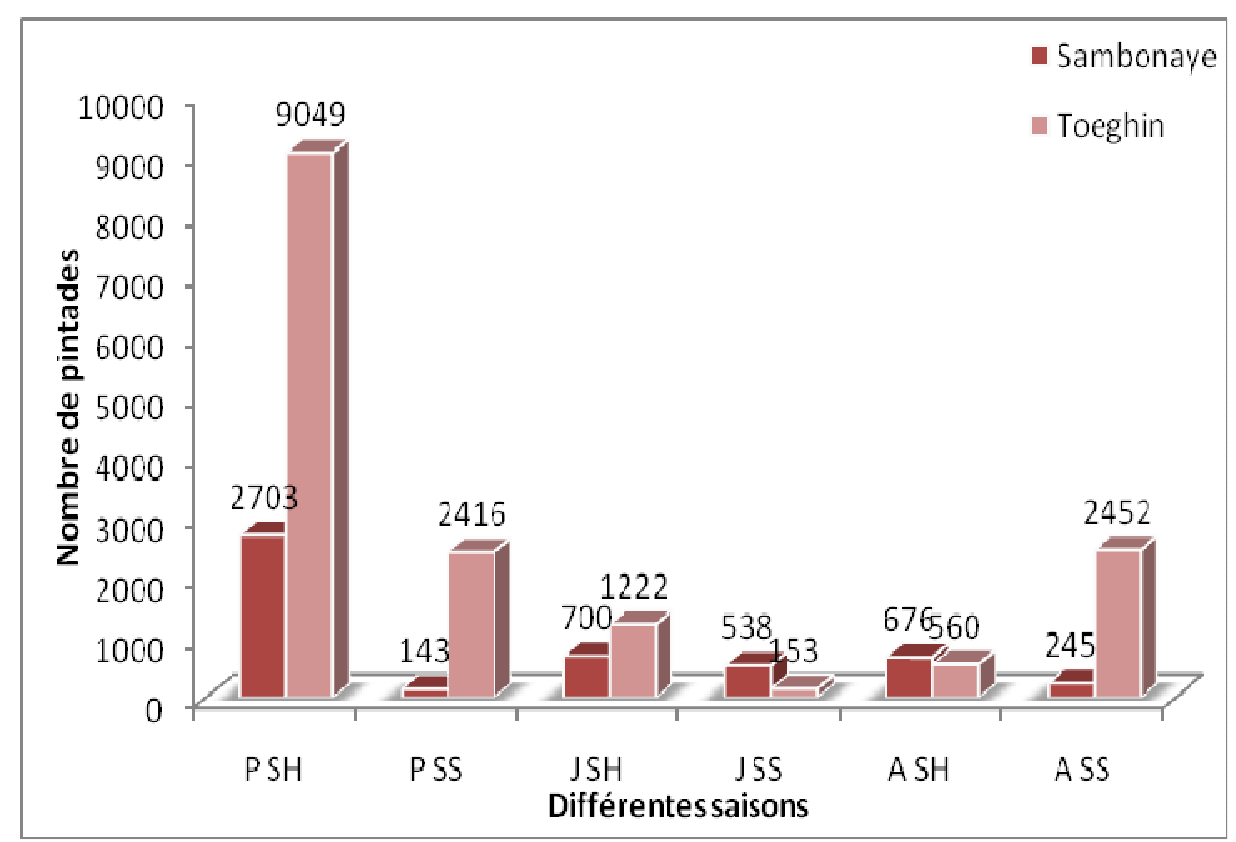

Figure 2: Effectif du cheptel de pintades de Sambonaye et de Toêghin selon la saison Pinta. Pintadeaux ; SH : Saison Humide ; SS : Saison Sèche.

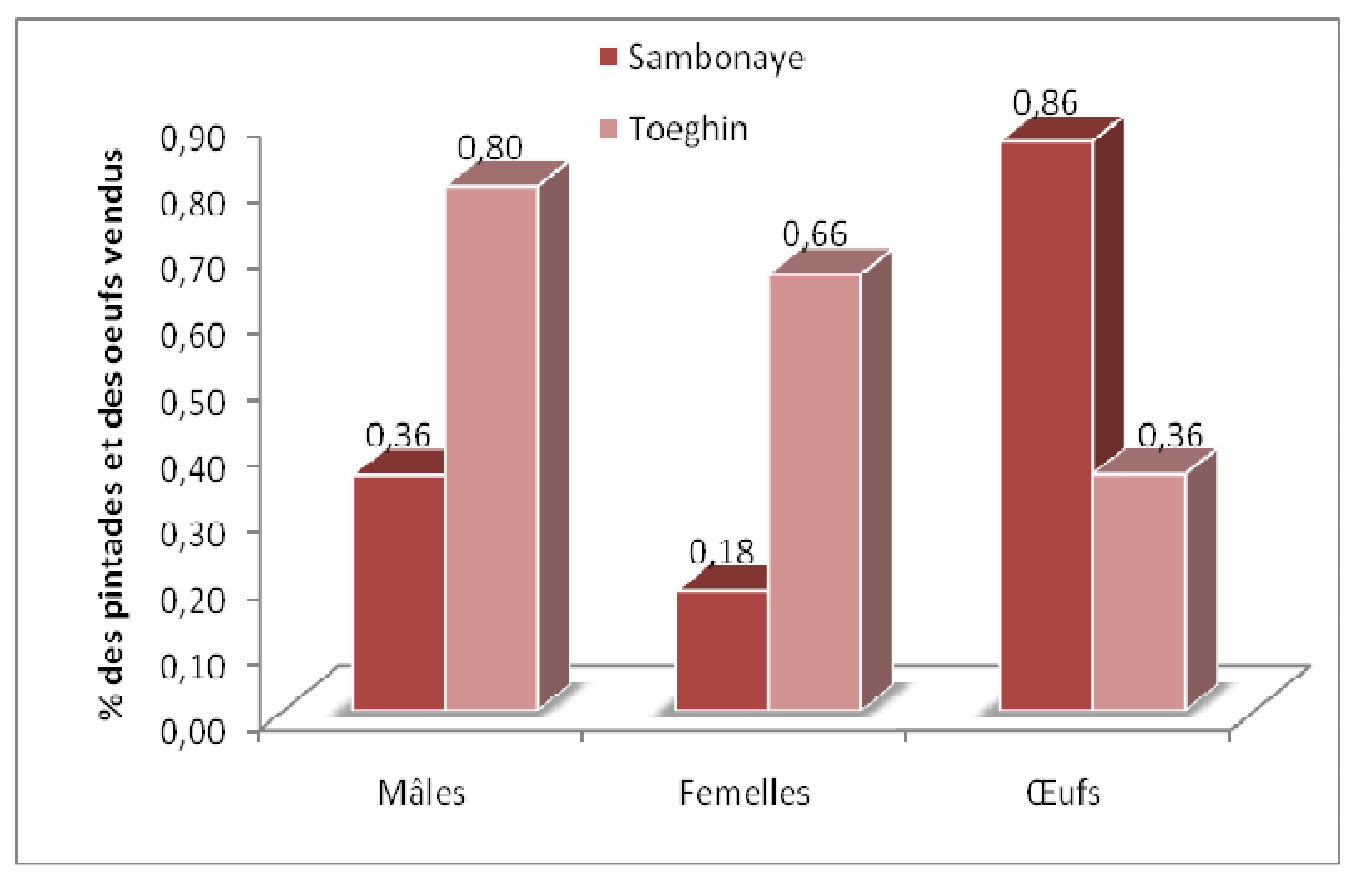

Figure 3: Pourcentage de pintades mâles et femelles et de leurs œufs vendus à Sambonaye et à Toêghin. 


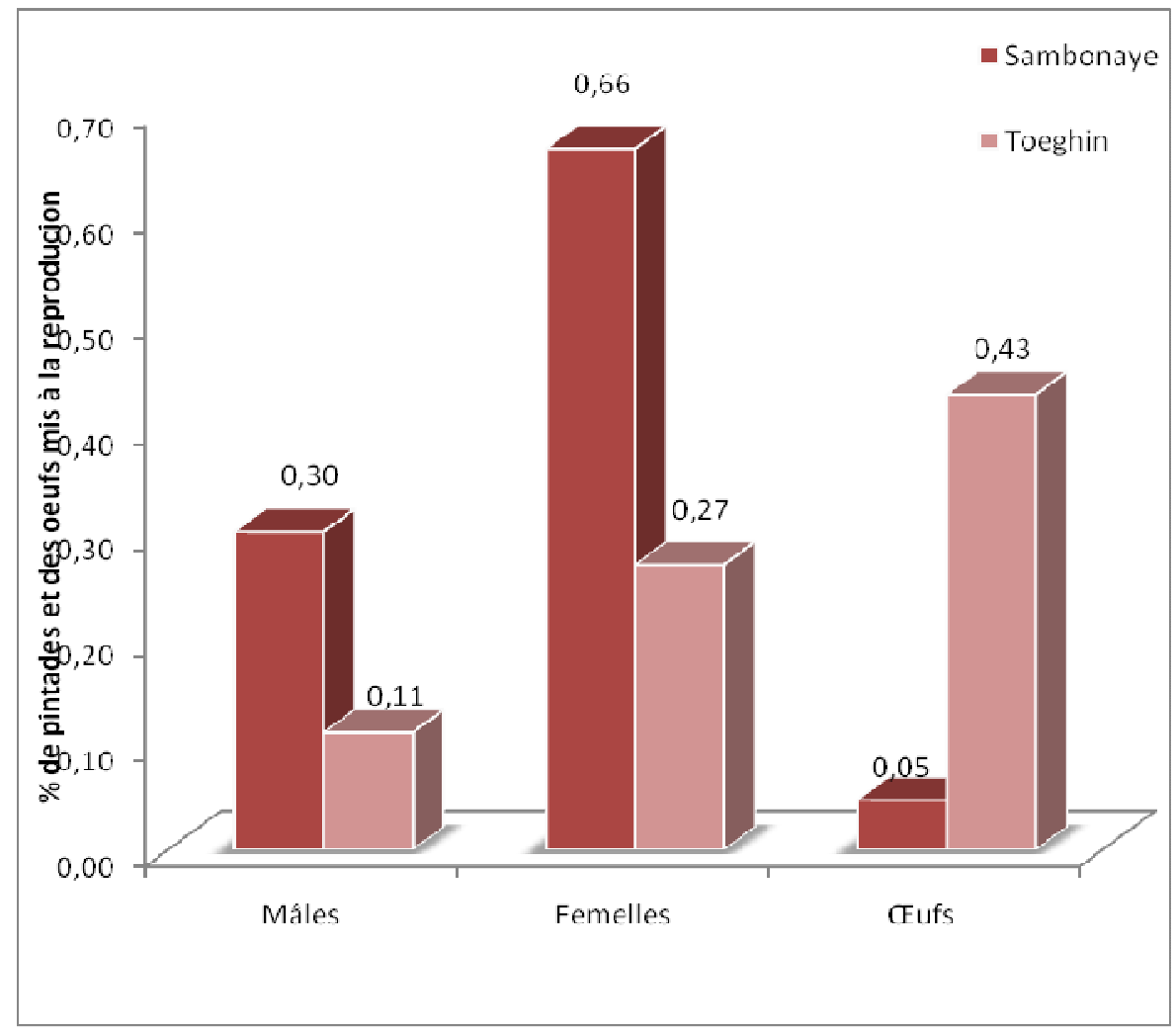

Figure 4: Pourcentage de pintades mâles, femelles et de leurs œufs mis à la reproduction.

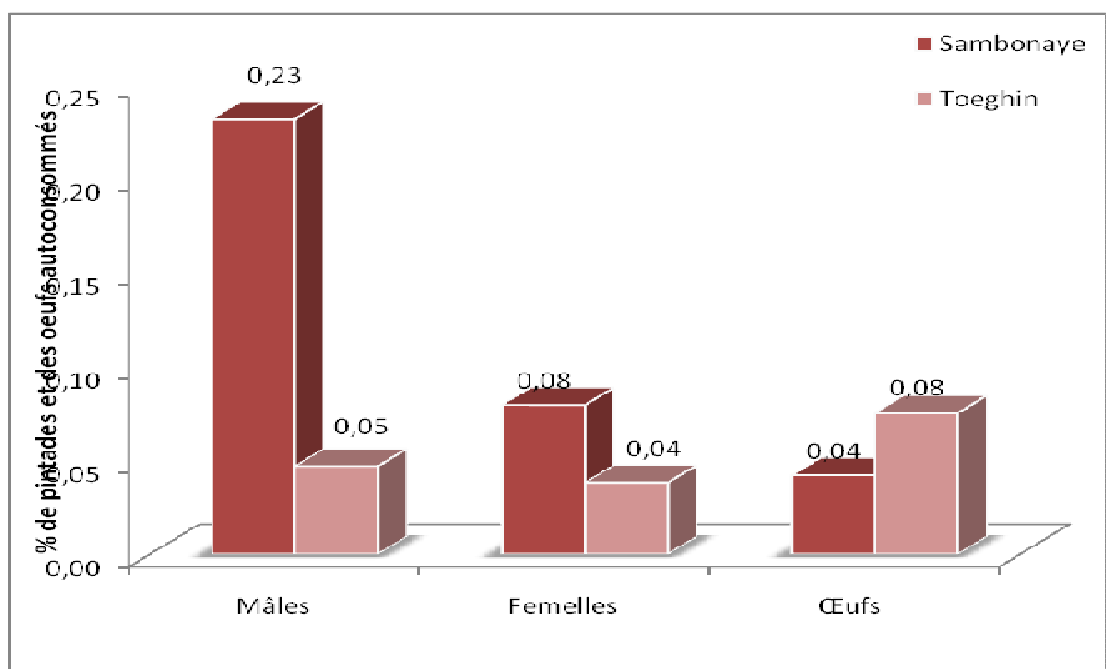

Figure 5: Pourcentage des Pintades mâles, femelles et de leurs œufs autoconsommés à Sambonaye et à Toêghin. 


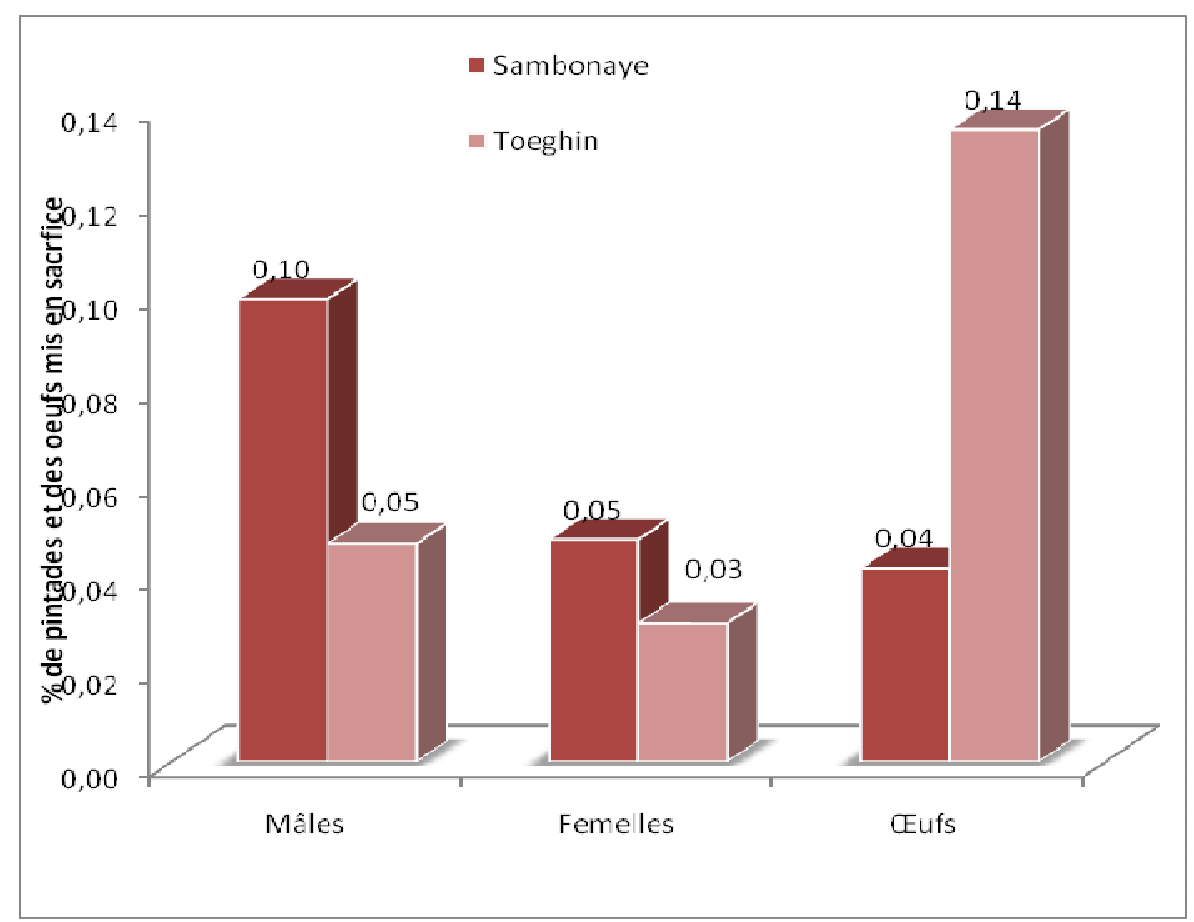

Figure 6: Pourcentage des pintades mâles, femelles et de leurs œufs exploités en dons à Sambonaye et à Toêghin.

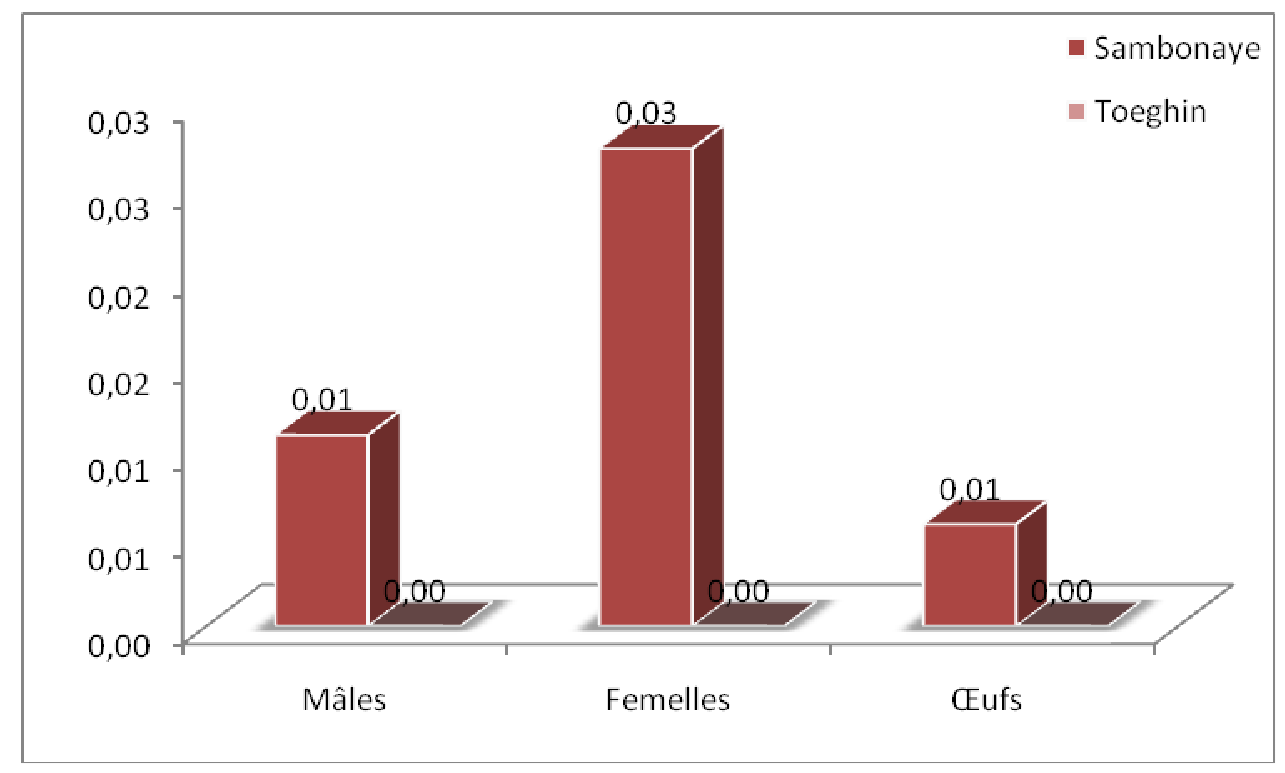

Figure 7: Nombre moyen de pintades mâles, femelles et des leurs œufs exploités sous forme de sacrifices par les producteurs de Sambonaye et de Toêghin. 
Tableau 4 : Valeurs en \% des pintades et de leurs œufs exploités dans les villages de Sambonaye et de Toêghin.

\begin{tabular}{|c|c|c|c|c|c|c|}
\hline \multirow[t]{2}{*}{ Exploitation } & \multicolumn{2}{|c|}{ Mâles (\%) } & \multicolumn{2}{|c|}{ Femelles (\%) } & \multicolumn{2}{|c|}{ Eufs(\%) } \\
\hline & \multicolumn{2}{|c|}{75,85} & \multicolumn{2}{|c|}{60,96} & \multicolumn{2}{|c|}{80,08} \\
\hline \multirow[t]{3}{*}{ Ventes } & Sambonaye & Toêghin & Sambonaye & Toêghin & Sambonaye & Toêghin \\
\hline & 3,01 & 72,84 & 1,99 & 58,97 & 78,01 & 2,07 \\
\hline & \multicolumn{2}{|c|}{12,72} & \multicolumn{2}{|c|}{31,32} & \multicolumn{2}{|c|}{9,37} \\
\hline \multirow[t]{3}{*}{ Reprod. } & Sambonaye & Toêghin & Sambonaye & Toêghin & Sambonaye & Toêghin \\
\hline & 2,54 & 10,18 & 7,28 & 24,04 & 4,26 & 5,11 \\
\hline & \multicolumn{2}{|c|}{6,24} & \multicolumn{2}{|c|}{4,26} & \multicolumn{2}{|c|}{4,69} \\
\hline \multirow[t]{3}{*}{ Autocon. } & Sambonaye & Toêghin & Sambonaye & Toêghin & Sambonaye & Toêghin \\
\hline & 1,95 & 4,29 & 0,87 & 3,39 & 3,80 & 0,89 \\
\hline & \multicolumn{2}{|c|}{5,10} & \multicolumn{2}{|c|}{3,16} & \multicolumn{2}{|c|}{5,33} \\
\hline \multirow[t]{3}{*}{ Dons } & Sambonaye & Toêghin & Sambonaye & Toêghin & Sambonaye & Toêghin \\
\hline & 0,83 & 4,27 & 0,52 & 2,64 & 3,73 & 1,60 \\
\hline & \multicolumn{2}{|c|}{0,09} & \multicolumn{2}{|c|}{0,30} & \multicolumn{2}{|c|}{0,53} \\
\hline \multirow[t]{2}{*}{ Sacrifices } & Sambonaye & Toêghin & Sambonaye & Toêghin & Sambonaye & Toêghin \\
\hline & 0,00 & 0,09 & 0,00 & 0,30 & 0,00 & 0,53 \\
\hline
\end{tabular}

\section{DISCUSSION}

Dans les deux villages, les femmes pratiquent l'élevage des pintades contrairement à celles de Villy, Namanguéma dans la Province du Sanguié, et Gampéla dans la Province du Kadiogo, où cette activité est exclusivement menée par les hommes de plus de 32 ans (Ojedapo et al., 2008). A Sambonaye et à Toêghin, l'âge n'est pas un facteur clé pour l'élevage des pintades. A partir de 23 ans, le producteur est habilité à élever des pintades. Ceci présente un avantage certain quant à la promotion de cette activité dans le milieu.

Les avantages et les inconvénients de l'élevage des pintades sont analogues à ceux trouvés par Nagalo (1984). Les dires des producteurs sont en accord avec ceux de Savadogo (1995), qui affirment que la forte mortalité des pintadeaux survient en saison pluvieuse, avec un pic au mois d'Août. Celleci peut atteindre de $73 \%$ à $100 \%$ (Savadogo, 1995 ; Bessin et al., 1998 ; Bako, 2004).
La mortalité des pintadeaux reste un des problèmes prédominants dans l'élevage de la pintade locale (Cirad-Iemvt, 1989 ; Bako, 2004 ; Guémené et al., 2001), ce qui réduit, voire anéantit les efforts des producteurs. Celle-ci est très élevée en hivernage, mais faible en saison sèche. Aussi, il est réconfortant de noter l'existence d'une période favorable à la survie des pintadeaux, qui offre une lueur d'espoir à la lutte contre leur mortalité.

Les prix des oiseaux ont fortement fluctué de 2007 à 2013 (Ojedapo et al., 2008). Ces prix ont doublé de 791,5 FCFA $(1,21 €)$ pour les jeunes mâles, de 755 FCFA $(1,15 €)$ pour les jeunes femelles, de 819 FCFA $(1,25$ $€)$ pour les adultes mâles et de 760,5 FCFA $(1,61 €)$ pour les adultes femelles. Quant aux prix des œufs, ils ont simplement augmenté de 16 FCFA $(0,02 €)$.

L'ordre dans l'exploitation de la pintade passe par les ventes, la reproduction, l'autoconsommation, les dons puis les sacrifices. Cette classification est conforme à 
celle de Sanfo (2007). La part de la vente dans l'exploitation révèle que la pintade est élevée essentiellement à but lucratif (MRA., 2003 ; Savadogo, 1995). L'inflation des prix des pintades et de leurs oufs constitue un facteur favorisant au passage des thèmes avicoles, surtout dans l'élevage des pintades, des chercheurs aux paysans et participe certainement, à court ou long terme, au développement de cette activité.

L'incubation des œufs reste un objectif prioritaire du producteur comme l'a également signifié Hien et al., (2001). Cependant, la part destinée à cette incubation est de loin inférieure à celle orientée vers la vente. Cette contradiction résulte de la faible capacité des moyens d'incubation actuellement disponibles par les producteurs, quoique ceux-ci y introduisent déjà 48,7\% de leurs meilleures poules mères à cette fin (Sanfo., 2002). L'utilisation des poules dans cette opération de couvaison draine deux conséquences majeures :

(i)- le faible nombre d'éclosions par couvée (Sanfo et Boly., 2002) qui influe sur l'efficacité de la gestion du cheptel ;

(ii)- le préjudice économique observé sur les poules couveuses et imputable au phénomène de l'incubation naturelle (Dembélé et al., 1996).

Ces conséquences de fond pourraient cependant être levées par l'introduction ou le développement de l'incubation artificielle dans l'aviculture villageoise. L'existence de couveuses de fabrication locale et de plus grande capacité (100 à 500 œufs) constitue un facteur favorisant. Le producteur pourrait augmenter la capacité de ses moyens d'incubation de 4 à 8 fois par l'introduction d'incubateurs, et améliorer par ailleurs les performances les taux d'éclosion. L'introduction et le développement de cette technologie devraient toutefois être soutenus par une formation et un encadrement des producteurs sur les conditions d'une bonne incubation (Ojedapo et al., 2008).

La pintade peut être impliquée dans les exploitations à titre alimentaire comme le poulet. Les pourcentages relevés par Hien et al. (2001) à ce titre sont de $15 \%$ pour les dons et $13 \%$ pour l'autoconsommation alors que le poulet est autoconsommé au taux de $25 \%$ et sortis sous forme de dons à 20\% (Bessin et al., 1998).

La faible ou la non implication de la pintade dans les événements sociaux, à Sambonaye et à Toêghin, tient de son caractère sauvage qui motive son exclusion des cérémonies rituelles (Cirad-Iemvt, 1989). La pintade peut toutefois accompagner le poulet dans ce domaine (Hien et al., 2001).

\section{Conclusion}

L'élevage de la pintade au Burkina Faso comporte à la fois des avantages et des inconvénients que le producteur de cette espèce rencontre dans l'activité qu'il mène. La mortalité des pintadeaux se révèle être l'un des problèmes majeurs qui inhibe l'action des producteurs. Par ailleurs, la pintade intervient dans les domaines alimentaires, et socioculturels. Le nombre de pintade évolue avec le temps, mais surtout en fonction de la saison sèche ou pluvieuse. Dans l'exploitation de la pintade, l'éleveur met l'accent sur la vente, la reproduction, l'autoconsommation, les dons et accessoirement les sacrifices. Le prix des pintades et des œufs ont subit une inflation de 2007 à 2013, ce qui laisse présager l'attraction de cette activité, à travers l'argent qu'elle procure, au niveau des producteurs de Sambonaye et de Toêghin. L'élevage quitte progressivement le chemin du social pour emprunter celui du lucratif. Celui-ci laisse présager que les producteurs seront à l'écoute des techniciens qui leur enseigneront les thèmes techniques sur l'élevage, et quitteront progressivement l'élevage traditionnel vers l'élevage moderne.

\section{REFERENCES}

Bako A. 2004. Stratégies d'élevage de pintadeaux dans la zone de BoboDioulasso: pertes économiques et moyens paysans de réduction de la mortalité. Rapport ATE. 42p.

Bessin NR, Belem AMG, Boussini H, Compaore Z, Kaboret Y, Dembélé MA. 
1998. Enquête sur les causes de mortalité des pintadeaux au Burkina Faso. Revue Élev. Méd.Vét. Pays Trop., 51(1): 87-93.

Cirad-Iemvt. 1989. Développement de l'aviculture traditionnelle en Afrique Tropicale. Ministère de la Coopération et du Développement. Fiche technique d'Elevage $\mathrm{N}^{\circ}$ 2. IEMVT. Maisons-Alfort -Jouve- Paris. France, 8 p.

Dembélé MP, Gnoumou H, Fréderic P. 1996. L'élevage de la pintade au Burkina Faso. Bulletin du Réseau Documentaire Élevage. $\mathrm{N}^{\circ} 4$ Spécial octobre.

Guémené D, Kansaku N, Zadworny D, 2001. L'expression du comportement d'incubation chez la dinde et sa maîtrise en élevage. INRA Prod. Anim., 14: 147160.

Hien OC, Nianogo AJ, Sawadogo L. 2001. L'élevage traditionnel de la pintade locale dans la zone centre-ouest du Burkina. Sciences et Techniques, 25(2).

MRA. 2003. Ministère des Ressources Animales. Méléagriculture au Burkina Faso : Diagnostic et axes d'amélioration. Secrétariat Général. Burkina Faso, 46 p.

Nagalo M. 1984. Contribution à l'étude du parasitisme chez la pintade commune (Numida meleagris) en Haute Volta, les helminthes parasites du tube digestif. Thèse : Méd. Vét. : Dakar. Sénégal, 9.112p.

Ojedapo Tossa I, Aboh AB, Houndonougbo MF, Gbego Tossa I, Kassavi E,
Chrysostome CAAM, Menshah GA. 2008. Influence du système d'élevage sur la performance de croissance pondérale au sevrage des poulets de l'écotype Fulani au Centre du Bénin. Tropical Animal Health and Production, 39(3): 155-64.

Sanfo R. 2007. Paramètres de reproduction de la pintade locale (Numida meleagris). Université de Ouagadougou. Thèse de doctorat, Burkina Faso, 129p.

Sanfo R. 2002. Performances et variabilité individuelle de la poule locale (Gallus gallus) au Centre - Ouest du Burkina Faso, Mémoire de DEA, 65p.

Sanfo R, Boly H. 2002. Productivité et variabilité individuelle du poulet local en conduite traditionnelle et améliorée au centre ouest du Burkina Faso. Mémoire de DEA. Institut du Développement Rural. Université Polytechnique de BoboDioulasso, Burkina Faso, 43 p.

Savadogo A. 1995. Contribution à l'amélioration de l'élevage de la pintade (Numida meleagris) au Burkina Faso. Mémoire de fin d'études d'IDR. Option : Elevage. Université de Ouagadougou, $102 p$.

Vidogbèna G, Addisu H, Zewdu W, Hailu M. 2010. Breeding practice and objective of indigenous chicken in North Wollo, Amhara regional State, Ethiopia. 8p. 